\title{
Integration of Current Clinical Knowledge with a Data Driven Approach: An Innovative Perspective
}

\author{
D. Mendes ${ }^{*}$, , S. Paredes ${ }^{\dagger}$, T. Rocha ${ }^{\dagger}$, P. Carvalho*, J. Henriques* \\ and J. Morais, \\ ${ }^{*}$ CISUC, University of Coimbra, DEI, Polo 2, Pinhal de Marrocos \\ Coimbra, 3030-290, Portugal \\ ${ }^{\dagger}$ Polytechnic Institute of Coimbra/ISEC, Rua Pedro Nunes - Quinta da Nora \\ Coimbra, 3030-199, Portugal \\ .Cardiology Department, Leiria Hospital Centre, Ruas das Olhavas \\ Leiria, 2410-197, Portugal \\ §diana.sxm@gmail.com
}

Published 5 December 2017

\begin{abstract}
Cardiovascular diseases are the leading cause of death worldwide. The development of models to support clinical decision is of great importance in the management of these diseases. This work aims to improve the performance exhibited by risk assessment scores that are applied in the clinical practice. This methodology has three main phases: (i) representation of scores as a decision tree; (ii) optimization of the decision tree thresholds using data from recent clinical datasets; (iii) transformation of the optimized decision tree into a new score.

This approach was validated in a cardiovascular disease secondary prevention context, supported by a dataset provided by the Portuguese Society of Cardiology $(N=13902)$. The respective performance was assessed using statistical metrics and was compared with GRACE score, the reference in Portuguese clinical practice. The new model originated a better balance between the sensitivity and specificity when compared with the GRACE, originating an accuracy improvement of approximately $22 \%$.
\end{abstract}

Keywords: Cardiovascular diseases; risk assessment; optimization; knowledge driven process; interpretability.

\section{Introduction}

Cardiovascular disease (CVD) is the general designation used to describe the disorders of the cardiovascular system that is responsible for supplying blood (oxygen, nutrients, etc.) to the entire body. ${ }^{1}$ Coronary artery disease (CAD), heart failure,

I On behalf of the investigators of the national registry on acute coronary syndromes, Portuguese Society of Cardiology

$\S$ Corresponding author.

This is an Open Access article published by World Scientific Publishing Company. It is distributed under the terms of the Creative Commons Attribution 4.0 (CC-BY) License. Further distribution of this work is permitted, provided the original work is properly cited. 
cerebrovascular disease and congenital heart disease are some examples of CVDs. According to World Health Organization (WHO), ${ }^{2}$ CVDs are the major cause of death worldwide, responsible for about $31 \%$ of global deaths in 2012 (more specifically, in Portugal this number is slightly higher reaching $32 \%$ of all deaths registered in that year $\left.{ }^{3}\right)$. Among the CVD, the $\mathrm{CAD}^{\mathrm{a}}$ assumes a great importance, as it was responsible for about 7.4 million deaths around the world.

It is recognized that the best strategy to reduce these numbers is to promote preventive health care. In this context, two categories of prevention are considered in clinical practice ${ }^{5}$ : primary prevention and secondary prevention. The former tries to minimize the probability of a healthy individual developing a disease while the latter is applied to patients with established disease, aiming to delay the disease progression. In order to help the clinician in his decision, there are guidelines that are followed in clinical practice, able to process the most significant patient's information. However, the assessment of a patient's condition is not always straightforward namely in the presence of a complex situation, when there are several sources of information to consider.

There are some models/scores used in clinical practice that were developed based on the analysis of large real patient datasets. Namely, in the context of secondary prevention, some of these models were specifically developed to predict the risk level of a patient suffering a new cardiovascular event (e.g., re-hospitalization or death). Particularly, in relation to CAD models, the Global Registry of Acute Coronary Events (GRACE), Thrombolysis In Myocardial Infarction (TIMI) and Platelet glycoprotein IIb/IIIa in Unstable angina: Receptor Suppression Using Integrilin (PURSUIT) are the most applied models in the Portuguese clinical practice. ${ }^{6}$ They were developed for short-term prognosis (30 days and 6 months) of a patient having a new cardiovascular event. These models (scores) consider a specific set of variables (risk factors) whose values can be split in categories, the final value results from the sum of the individual risk factors. The global value is compared with thresholds that allow the identification of several groups of risk (e.g., low, intermediate and high). Although useful, these models have some limitations, namely: they are not prepared to deal with missing information, they cannot cope with the incorporation of new knowledge (e.g., a new risk factor) and they might present some problems when applied to a specific patient. ${ }^{7}$

Additionally, these models do not take into account the evolution in the clinical interventions and treatments verified during the last years. Moreover, the information related with each patient (collected both in hospitals and/or through telemonitoring systems) is currently stored in clinical datasets. However, this information is frequently disregarded. Therefore, the development of innovative

\footnotetext{
${ }^{a}$ CADs include all the problems related with artery narrowing in the coronary vessels. This disease can be characterized through two different conditions. ${ }^{4}$ (i) Acute coronary syndromes (unstable angina/non-Q wave Myocardial infarction) defined by a transient occlusion; (ii) Myocardial infarction that is defined by a complete occlusion and tissue necrosis.
} 
methodologies, able to update risk scores using information stored in recent clinical datasets, is decisive in improving and increasing the confidence of the physician's decision. As a result, the main goal is to develop methodologies to improve the management of patient's risk scores, supporting the clinical decision in order to reduce the occurrence of undesired events. The proposed approach is based on two main sources of information: (i) the information provided by the models already used in clinical practice (knowledge driven approach); (ii) the analysis of recent datasets (data driven approach). In effect, the development of algorithms to infer knowledge from data and mechanism to support its integration and application with the current clinical evidence in daily clinical practice will definitely contribute to improve the decision process. The approach is depicted in Fig. 1, defined by three main phases:

Phase $\mathbf{A}$ addresses the representation of the existing clinical knowledge, in this case risk tools; Phase $\mathbf{B}$ deals with knowledge discovery strategies, able to extract useful new knowledge from existent clinical datasets; Phase $\mathbf{C}$ involves flexible and adequate hybrid combination schemes, that allow the combination of the new knowledge with the current risk assessment models in order to improve the clinical decision. The proposed approach intends to improve risk assessment tools. In particular, the way the recent clinical datasets can be used to optimize the existing models (e.g., GRACE) is explored.

The GRACE score is the most well accepted model in Portuguese clinical practice in the context of secondary prevention applied to CAD patients. ${ }^{8}$ Therefore, this score was selected as the reference in this work. It involves information of eight risk factors (age, systolic blood pressure, heart rate, creatinine, killip class, ST-segment deviation, elevated cardiac markers and cardiac arrest at admission).

Several attempts have been made to improve GRACE score. In 2011, de Mulder et $a l .{ }^{9}$ investigated whether the admission of glucose could improve the GRACE

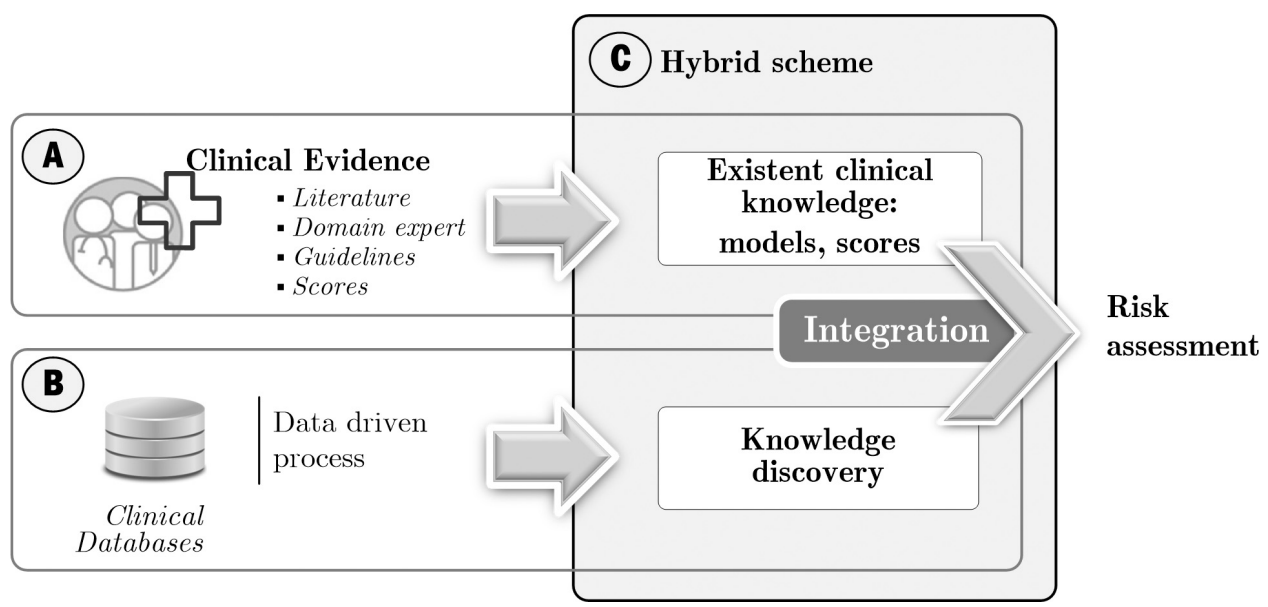

Fig. 1. Global strategy. 
score assessment, as in the hospital admission the plasma glucose is a biomarker of prediction of mortality in myocardial infarction. Data from 550 patients admitted from 2003 to 2006 in care unit of Medical Center Alkmaar was applied. The GRACE score was calculated for every patient. Then a logistic regression model was created using this score to compute the probability of death for each patient with and without glucose. The results were presented through the values of area under the ROC curve and showed that the introduction of glucose did not increase the predictive capacity of the GRACE score. In 2014, Fox et al. ${ }^{10}$ developed a more accurate version of GRACE risk score. The study included 32,037 patients (14 countries, 94 hospitals) collected during 10 years for the training phase. This methodology focused on nonlinear associations for continuous risk factors. A French registry of Acute ST elevation and non-ST elevation MI (FAST-MI) 2005 was applied for the validation procedure. The patient's risk was assessed for 1, 2 and 3 years and the output was given as absolute percentage risk. Besides the nonlinear association in GRACE score, the authors introduced a solution for cases where the features creatinine and Killip class are not available. In those cases, these features were replaced by diuretic usage and renal failure, respectively. The authors concluded that the new model is easier to apply than the original GRACE as well as presenting a better performance when compared with the original model. Recently, Paredes et al. ${ }^{7,11}$ developed a methodology in order to minimize some weaknesses of actual models used in clinical practice (TIMI, GRACE, PURSUIT). The proposed approach had two main components. In the first, the individual risk models were subjected to fusion through a common representation (by applying the Naive Bayes classifier). In the second approach, a personalized strategy was developed, and for each patient the most suitable risk tool was considered. A real dataset collected in Santa Cruz Hospital (Lisbon, Portugal) was used for train and validation. In terms of results, both methodologies presented better performance compared with the individual risk scores.

The current paper explores the application of machine learning techniques ${ }^{12-15}$ to update the GRACE score. In particular, decision trees (DTs) are used to extract and represent knowledge ${ }^{16,17}$ from recent clinical datasets, as well as to describe the score. In fact, DTs are a key element, as they assure the clinical interpretability of the model (one of the major aspects in the clinical domain) and they organize the information in rules, which seems particularly well adapted to describe the score as well as the new knowledge. In this work, a dataset from the Portuguese Society of Cardiology (PSC) was considered for validation purposes. This dataset comprises information of 13902 patients, collected in the context of the second stage of the national registration of acute coronary syndrome, gathered between 1st October 2010 and 26th February 2016.

In Sec. 2, the proposed methodology is detailed. Section 3 contains a brief description of the dataset as well as the main results; Section 4 focuses on the discussion of obtained results; finally, Sec. 5 focuses on the main conclusions as well as on the identification of guidelines for ongoing research. 


\section{Methodology}

Figure 2 systematizes the methodology followed during this work. Three important phases can be identified: (1) representation of a score through a set of rules (using DTs); (2) optimization of the rules, more specifically, modifying the thresholds $\left(T_{i}\right)$ in each rule (maintaining the structure of the tree); and (3) derivation of a new version of the original score based on the optimization performed in the DT.

\subsection{Representing the score as a decision tree}

$\mathrm{DTs}^{18}$ are tree-like graphs that illustrate the relationships or dependencies among risk factors. Branches exhibit the relations between nodes (that represent the risk factors), while leaves represent class labels. ${ }^{19}$ Due to their simplicity and structure, DTs are easily interpreted. Consequently, given these characteristics, DTs seemed adequate to describe a score assuring its clinical interpretability. Here, the DT is developed based on an artificial dataset. It is created semi-randomly taking into account the original risk factors' distribution, the mean value and standard deviation for continuous values and the incidence rate for binary values. All the instances are classified by a risk model currently applied in the clinical practice (score). This output is assumed as the real output (true data), allowing in that way, the replication of the score's behavior by the DT. In relation to DT development, the classification and Regression Tree (CART) algorithm ${ }^{20-22}$ was implemented. CART generates a binary tree through several binary splits of data. The data are recursively split in two subsets based on risk factors, according to the minimization of a heterogeneity criterion (the Gini's diversity index ${ }^{23,24}$ in this work). The derivation of the DT is implemented through the Statistics and Machine Learning, toolbox from MATLAB.

\subsection{Optimization algorithm}

There are several algorithms developed for optimization problems. ${ }^{25-28}$ In some particular cases, an analytical methodology can be followed although due to the usual complexity of the optimization problems, most of the methods are based on heuristic and/or iterative approaches. In this work, given its specific formulation, an iterative approach is followed, where the thresholds of the DTs are iteratively

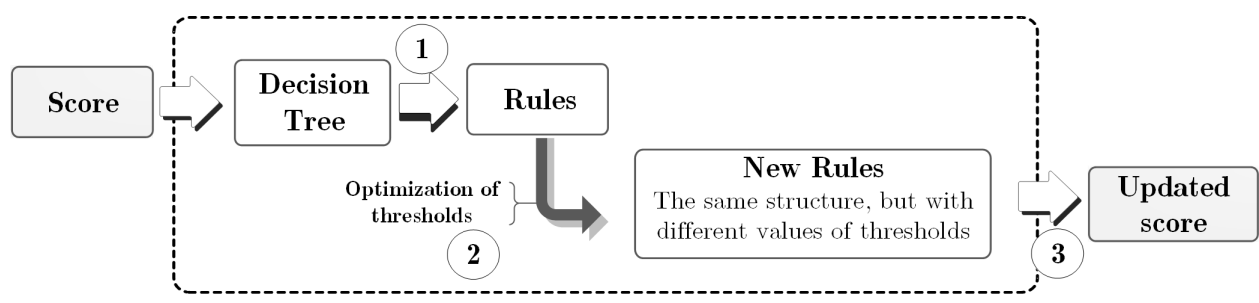

Fig. 2. Optimization (new approach). 
optimized based on dependencies among risk factors. In particular, the method takes into account the membership degree of each patient to each rule in order to adjust the thresholds.

\subsubsection{Main functions}

The developed algorithm intends to optimize the thresholds in each rule, without changing the original tree structure, i.e., keeping the dependencies among risk factors and the consequent output of these relations. Two different functions are the key elements of the proposed algorithm: the error function (EF) and the membership function $(\mathrm{MF})$.

\subsubsection{1. $\mathrm{EF}$}

The EF is defined as the module of the difference between two outputs, the actual event extracted from the dataset $\left(Y_{\text {real }}\right)$ and the estimated output obtained based on the DT ( $\left.Y_{\text {estimated }}\right)$, as described in Eq. (1).

$$
\mathrm{EF}=\left|Y_{\text {real }}-Y_{\text {estimated }}\right|
$$

This function quantifies the need for an adaptation of the thresholds $\left(T_{i}\right)$ considering each one of the patients. In the case of binary outputs, where the $Y_{\text {estimated }}$ and $Y_{\text {real }}$ only assume two values, 0 or 1, if the estimated output is equal to the actual one, the EF presents 0 value. In this case, it means that the thresholds do not need to be modified. On the other hand, if the EF value is equal to 1, the thresholds should be modified.

\subsubsection{2. $\mathrm{MF}$}

The MF gives information about the degree of membership of each patient in relation to a given condition in a node (each node in the DT represents a condition). Additionally, the MF is computed in all nodes that belong to each rule. The final value for a given rule $\left(\mathrm{MF}_{R}\right)$ is computed as the average of the $\mathrm{MF}_{T_{i}}$ present in each condition, Eq. (2), while $N$ is the number of conditions present in a specific rule (see Fig. 3).

$$
\mathrm{MF}_{R}=\frac{\sum_{i=1}^{N} \mathrm{MF}_{T_{i}}}{N} .
$$

In the particular case of rule 3

$$
\text { rule } 3: \text { if }(X 1<T 1) \quad \text { AND } \quad(X 2 \geq T 2) \quad \text { AND } \quad(X 4<T 4) \quad \text { THEN } y=0
$$

depicted in Fig. 3, the final MF function is computed taking into account the $M F$ of each specific condition $(\mathrm{MF}=a, \mathrm{MF}=d, \mathrm{MF}=g)$, i.e., the result of each comparison $(X 1<T 1),(X 2 \geq T 2)$, and $(X 4<T 4)$, with $N=3$.

For binary values, i.e., when the risk factors assume only two values ( 0 or 1$)$, the $\mathrm{MF}_{T_{i}}$ is given by Eq. (3). As a result, the patient belongs to a given condition 


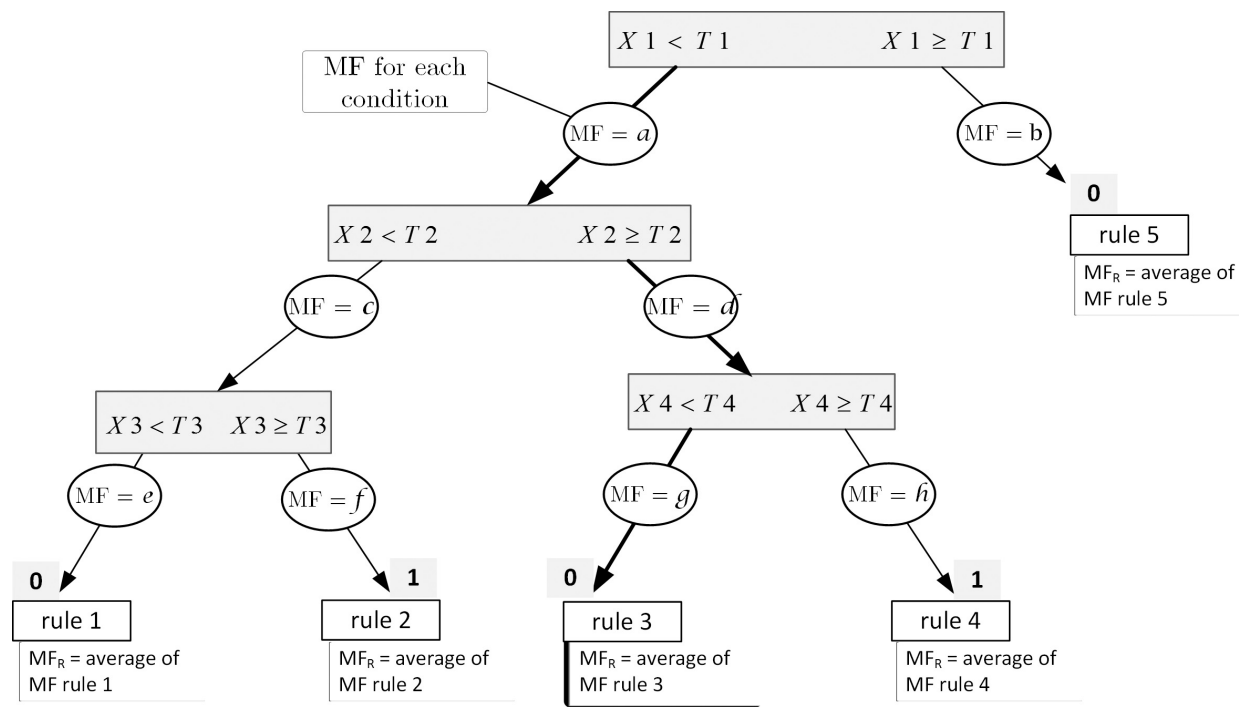

Fig. 3. MF values (example).

$\left(\mathrm{MF}_{T_{i}}=0\right)$ or not $\left(\mathrm{MF}_{T_{i}}=0\right)$.

$$
\mathrm{MF}_{T_{i}}= \begin{cases}1 & \text { if the condition is verified } \\ 0 & \text { if the condition is not verified. }\end{cases}
$$

In the case of continuous risk factors, the degree of membership should reflect a continuous variation. For example, let us consider a specific condition, $\left(X_{i}>T_{i}\right)$, describing the age of a patient (age greater than 50). With this condition, for a 55 year old patient, the MF value for a binary or a continuous formulation should be 1 . However, if a patient is 48 year old, the MF value for a continuous formulation should not be equal to zero, since the real age of the patient (48) is approximately equal to the threshold of the condition, $T_{i}=50$ (for a binary formulation the MF is, obviously, zero).

In order to address this issue, the sigmoid function (SF) was considered (Fig. 4) to define the range of the degree of membership in continuous risk factors, Eq. (4). The value of $\alpha$ can be used to parametrize the sigmoid (define the slope and the support of the sigmoid, i.e., values that are clearly distinct from 0 and 1 ), and $C$ introduces a time shift.

$\mathrm{MF}_{T_{i}}= \begin{cases}1 & \text { if the condition is verified and the } \\ 0 & \text { if the condition is not verified and } \\ & \text { binaries } \\ \frac{1}{1+e^{\alpha(-x+C)}} & \text { if the risk factors are continuous. }\end{cases}$ 


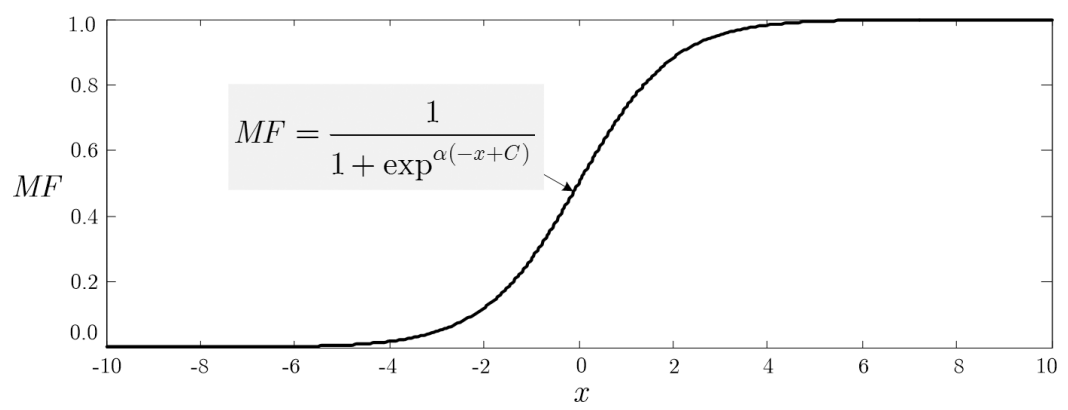

Fig. 4. Sigmoid function $(\alpha=1, C=0)$.

Following the same reasoning used in binary risk factors, a MF value equal to 1 , should be achieved when the patient verifies a condition $\left(X_{i}>T_{i}\right)$. However, using a sigmoid function, the MF never reaches the value 1 . Therefore, a lower value had to be considered for the MF value when the condition limit is verified, i.e., $X_{i}=T_{i}$. The value $\mathrm{MF}=0$, with $\theta=0.9$ can be assumed as an acceptable value. In order to reflect this assumption, the value of $C$ has to be determined in order to verify Eq. (5).

$$
\mathrm{MF}=\theta=\left.\frac{1}{1+e^{\alpha(-x+C)}}\right|_{x=T_{i}} .
$$

As result of Eq. (5), a distinct value of $C$ is computed for each condition (each node of the tree), according to Eq. (6).

$$
C=\frac{1}{\alpha} \ln \left(\frac{1-\theta}{\theta}\right)+T_{i}
$$

Moreover, the shape of the sigmoid (that defines the support, i.e., values greater than 0 and lower than 1) should also be defined according to the specificity of the problem. In this work, in order to preserve the same slope for all membership functions Eq. (7) was applied. The variable $R$ defines the range of the interval considered for each risk factor, and the value $\omega$ is an adequate normalization factor.

$$
\alpha=\frac{1}{R / \omega} \text {. }
$$

For example, for the sigmoid presented in Fig. 4, since the possible interval is $\omega \in[-10,10]$, the range is $R=21$. In this case, the normalization factor $\omega=21$ was employed resulting in $\alpha=1$

In a DT, each node provides two different options, which lead to two quite different conditions:

- Path $1-\left(X_{i} \geq T_{i}\right)$ - patient's value $(X i)$ higher or equal to the threshold $\left(T_{i}\right)$; - Path 2 - $\left(X_{i}<T_{i}\right)$ - patient's value lower than the threshold.

These two paths are here distinguished using a variable signal: the path1 assumes the signal with value 1 (signal $=1)$, while in path2 the signal is equal to -1 
( signal $=-1)$. For MF involving binary values, this signal is introduced in the MF as given by Eq. (8).

$$
\mathrm{MF}_{T_{i}}=\left\{\begin{array}{cc}
1 \quad & \text { if }\left(x<T_{i} \wedge \text { signal }=-1\right) \vee\left(x>T_{i} \wedge \text { signal }=1\right) \text {, for binary } \\
& \text { risk factors } \\
0 \quad & \text { if }\left(x>T_{i} \wedge \text { signal }=-1\right) \vee\left(x<T_{i} \wedge \text { signal }=1\right) \text {, for binary } . \\
& \text { risk factors. }
\end{array}\right.
$$

In relation to continuous values, the characterization of the two paths has to be distinct. For instance, considering the following condition, the heart rate is greater or equal than 100 , thus $\left(X_{i} \geq T_{i}\right)$, with $T_{i}=100$. Moreover, according to Eqs. (5) and (6), for $X_{i}=T_{i}=100, \mathrm{MF}=\theta$. If a given patient presents a heart rate equals to 105 (e.g., $X_{i}=105$ ), the condition is verified. Therefore, according to the above definition, the MF value should be higher or equal to $\theta$. On the other hand, in case the patient's heart rate is lower than $T_{i}$ (e.g., $X_{i}=95$ ), the condition is not verified. However, since this value (95) is close to the threshold (100), a MF value slightly lower than $\theta$ should be verified (Fig. 5).

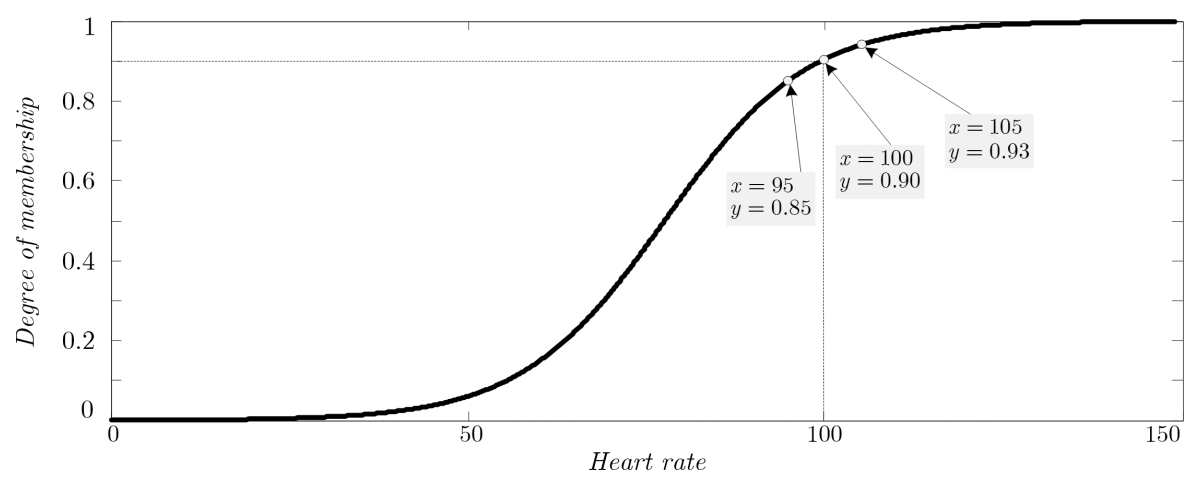

Fig. 5. Sigmoid function when signal $=1(\alpha=0.9)$.

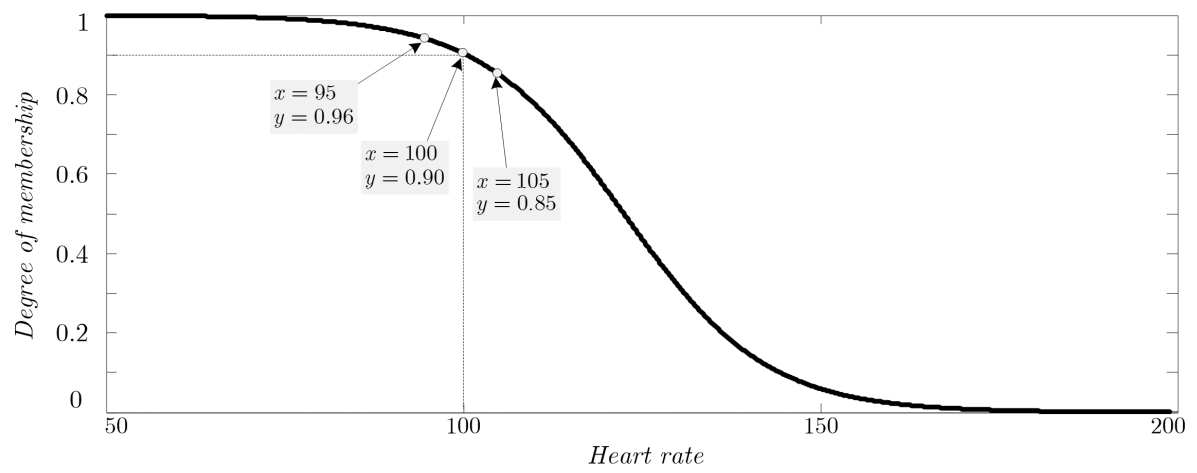

Fig. 6. Sigmoid function when signal $=-1(\alpha=0.9)$. 
On the other hand, the corresponding condition for the path2 $\left(X_{i}<T_{i}\right)$, can be described as heart rate is lower than 100. In this case, if a patient presents a heart rate equal to 95 , he verifies the condition $(95<100)$ and, as mentioned, the MF value should be higher than $\theta$. If a patient presents a value higher than the threshold (e.g., $X_{i}=105$ ), the reasoning is similar to the previous case. In this case the MF value should be lower than $\theta$ (see Fig. 6).

As conclusion, to deal with continuous membership functions and different behaviors (signal-1 or 1) Eq. (8) is updated, resulting in Eq. (9).

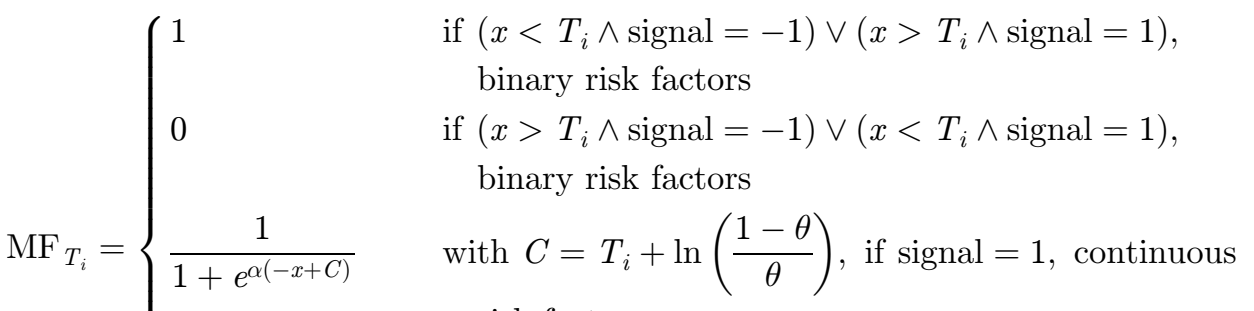

$$
\begin{aligned}
& \text { risk factors }
\end{aligned}
$$

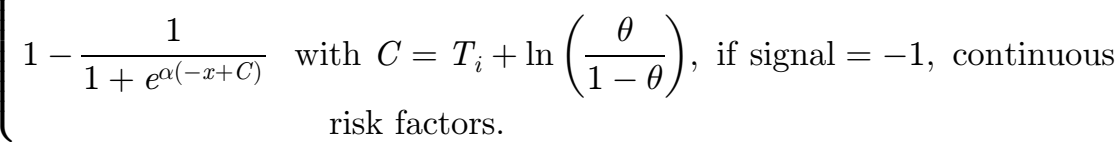

\subsubsection{Updating of the thresholds}

The optimization process (updating the thresholds $-T_{i}$ ) is performed iteratively, for each iteration $k$, and takes into account the values of membership (MF $\in \mathbb{R}^{n P \times n R}$ ) of each patient for each rule, as well as the respective error function $\left(\mathrm{EF} \in \mathbb{N}^{1 \times n P}\right)$. The variable $n R$ is the number of rules and $n P$ is the number of patients.

In the first step, a matrix composed of signals $\{-1,1\}, S R \in \mathbb{Z}^{n R \times n T}$, where $n T$ defines the number of thresholds, was generated (matrix SR). The signals $\{-1,1\}$, represent, respectively the conditions $\left(X_{i}<T_{i}\right)$ and $\left(X_{i} \geq T_{i}\right)$ that compose the DT. Moreover, the frequency that each node is employed to compute the output of the DT (the risk of the patient) should be taken into account. In effect, there are some nodes that are used more frequently than others, thus with a different contribution to the final risk. Therefore, to deal with this circumstance, a normalization factor was added in order to avoid the over-evaluation. As a result, the inverse of the number of times a node $\left(1 / \mathrm{N}, N \in \mathbb{N}^{1 \times n T}\right)$ was applied to adjust the computation of each threshold. Finally, to deal with possible instabilities resulting from higher adjustments, a gain factor $\mathrm{G}$ (scalar) was incorporated into the adjustment of the thresholds $(\triangle T)$, originating Eqs. (10) and (11).

$$
\begin{gathered}
\triangle T(k)=\frac{G \times \mathrm{EF} \times \mathrm{MF} \times \mathrm{SR}}{N}, \quad \triangle T \in \mathbb{R}^{1 \times n T}, \\
T(k)=T(k-1)-\triangle T(k)
\end{gathered}
$$


In order to guarantee that the updated values belong to an appropriate range (i.e., values with clinical significance), an allowed variation $(V 0)$ is defined as shown in Eqs. (12), (13) and (14). The new values range from LB to UB (respectively, lower bound and upper bound) and $\beta$ is a gain that represents a percentage of change of each parameter.

$$
\begin{gathered}
V 0=\beta \times T_{i}(k=0), \\
\mathrm{LB}=T_{i}(k=0)-V 0, \\
\mathrm{UB}=T_{i}(k=0)+V 0 .
\end{gathered}
$$

The iteration process runs until a pre-defined number of iterations is achieved. Then, the set of thresholds that attains the best geometric mean value suffers a new variation, according to Eq. (15), where the variable $\delta$ is a gain factor and the rand represents a random value between 0 and 1 .

$$
T_{m}(k)=T(k)+\delta(\text { rand }-0.5) .
$$

This variation is introduced as an attempt to avoid local minimums. Following this procedure, this new set $\left(T_{m}\right)$ is used as initial thresholds for a new optimization process (new cycle of iterations).

\subsection{Decision tree to score}

The final phase intends to derive a score taking into account the optimized DT. The goal is to generate a score through a set of rules capable to describe the patients according to the original score. The main advantage is related with the possibility of providing a score to the physicians that are familiar with (e.g., GRACE) and at the same time to improve its interpretability (described by a set of rules, supported on a DT).

The approach to achieve this goal takes into account how the risk factors are organized in the tree. For each risk factor, the correspondent thresholds are registered and organized in ascending order, with the aim of creating several ranges for each risk factor. Once the ranges for all the risk factors $(i)$ are stored, the rate of patients with high risk (sc) in each interval $(j)$ was computed through Eq. (16).

$$
\mathrm{sc}_{i_{j}}=\frac{\mathrm{Nhr}_{i_{j}}}{\mathrm{Nhr}_{i_{j}}+\mathrm{Nlr}_{i_{j}}} \times 100 .
$$

The variables Nhr and Nlr correspond to the number of patient with high and low risk for each interval of each characteristic. The variable $s c$ is seen as the score value associated to each category. Similarly, to a regular score, the value assigned to each category of each risk factor is added and the patients are classified as high risk or low risk taking into account the global value and the respective decision threshold. 


\section{Results}

\subsection{Portuguese society of cardiology dataset}

The PSC dataset contains information of 13,902 patients collected in the context of the second stage of the national registers of acute coronary syndrome, between 1st October 2010 and 26th February 2016. A pre-processing of data was performed. Patients with the same characteristics (same categories in all the considered risk factors) that present different behaviors (event/no event) were removed. Therefore, the dataset was reduced to 10,102 patients with an events rate of $3.98 \%$.

Regarding the validation procedure, the dataset was split in two phases: (i) Optimization of thresholds of the derived DT; (ii) - Development of a new score. Initially, the dataset was restricted to the patients collected between 1st October 2010 and 6th November 2014 (7,717 patients). This dataset was designated as set 1 and it was applied in both phases. Recently, the access to patients collected between 7th November 2014 and 26th February 2016 (2385 patients) was provided. This dataset designated as (set 2) was exclusively used for the validation of the phase (ii).

The characterization of the complete dataset was performed based on GRACE's risk factors. Table 1 shows the values of the different characteristics for the sets 1 and 2 , respectively. In the case of continuous variables, the mean value and the standard deviation are presented, while in relation to binary values the incidence rate is depicted.

Missing information was also one of the problems addressed in this phase, as some patients presented incomplete information. In order to deal with this problem (missing values), statistical imputation methods were adopted. ${ }^{29}$ For continuous risk factors, the average of the risk factor's values that correspond to the missing value was applied, while the most common value was used in relation to binary risk factors.

\subsection{GRACE score}

As already mentioned, the $\mathrm{GRACE}^{30}$ score was selected as the reference to the validation procedure. This score ${ }^{31}$ involves information from eight risk factors that

Table 1. Characteristics on admission of the patients with acute coronary syndromes (GRACE score features) - Train and Test set. The nd value refers to the rate of missing values.

\begin{tabular}{lcc}
\hline Model & Set 1 & Set 2 \\
\hline Age (years|nd) & $66.06 \pm 13.86 \mid 0.0001$ & $66.39 \pm 13.38 \mid 0.0004$ \\
Creatinine $(\mathrm{mg} / \mathrm{dL} \mid \mathrm{nd})$ & $1.23 \pm 1.26 \mid 0.0804$ & $1.16 \pm 1.06 \mid 0.0243$ \\
Systolic Blood Pressure (mmHg|nd) & $138.32 \pm 31.81 \mid 0.0034$ & $139.81 \pm 29.82 \mid 0.0013$ \\
Heart Rate (bpm|nd) & $78.84 \pm 21.88 \mid 0.0131$ & $77.80 \pm 20.52 \mid 0.0109$ \\
Killip class $(1 / 2 / 3 / 4 \mid \mathrm{nd})$ & $0.7960 / 0.1171 / 0.0563 / 0.0260 \mid 0.0045$ & $0.8415 / 0.0998 / 0.0348 /$ \\
& & $0.0210 \mid 0.0029$ \\
ST-segment deviation $(1 / 0 \mid \mathrm{nd})$ & $0.8134 / 0.1866 \mid 0$ & $0.8289 / 0.1711 \mid 0$ \\
Elevated cardiac markers $(1 / 0 \mid \mathrm{nd})$ & $0.9015 / 0.0985 \mid 0.0001$ & $0.9312 / 0.0688 \mid 0$ \\
Cardiac arrest at admission $(1 / 0 \mid \mathrm{nd})$ & $0.0275 / 0.9725 \mid 0$ & $0.0197 / 0.9803 \mid 0$ \\
\hline
\end{tabular}


are presented in Table 1. According to this score, each risk factor is split in categories and each category has a given value of risk. ${ }^{32}$

In order to assess the risk level of a patient, a specific value according to the GRACE categorization is given to each one of the respective inputs (risk factors), for example if the patients heart-rate is in the interval [110-149] the respective GRACE value is 21 (Table 8). Then, the numerical global risk is computed through the sum of these individual GRACE values. Finally, this sum is converted to a risk level (such as low, medium, high) according to the ranges defined in Table $2 .^{33}$

The PSC dataset only has information about two conditions: if the patient suffers a new event (high risk) or not (low risk). In this way, there is no information about the intermediate state. Thus, the thresholds of original score had to be adapted for two levels of risk in this work. The low risk includes the ranges of low and intermediate risk of Table 2 and the high risk does not suffer any modification.

\subsection{Decision tree}

In order to avoid the overfitting to the PSC dataset, the DT was developed using an artificial dataset composed by 4,000 observations (balanced dataset - 2000 observations classified as no event and 2,000 observations classified as event). The artificial dataset was constructed semi-randomly, that is, the values for each risk factor were attributed randomly but ensuring that the distribution of each risk factor is similar with its distribution in the PSC dataset (mean value and standard deviation in the case of continuous risk factors and the incidence rate related with binary risk factors). As mentioned, this dataset was required to develop a DT capable to reproduce the original score behavior. In this situation, the output provided by the GRACE score was considered the real output (true data).

Therefore, the DT was developed using this artificial dataset and taking into account the respective GRACE output. The generated DT was composed by a significant number of rules and each rule was composed by several nodes, which make these rules hard to interpret and difficult to apply in clinical practice. Therefore, the reduction of the number of rules was applied to minimize this flaw based on a pruning method (cost-complexity pruning). ${ }^{20}$ The branches that cause less improvement in error cost were removed and replaced by a leaf node whose value is chosen based on Gini's diversity index. The best results (five possible alternatives) of the DTs are presented in Table 3.

Table 2. Range of risk according to GRACE score.

\begin{tabular}{|c|c|c|c|}
\hline \multicolumn{2}{|c|}{ Non STE-ACS: In-hospital mortality } & \multicolumn{2}{|c|}{ STE-ACS: In-hospital mortality } \\
\hline$<109$ & Low risk & $<126$ & Low risk \\
\hline $109-140$ & Intermediate risk & $126-154$ & Intermediate risk \\
\hline$>140$ & High risk & $>154$ & High risk \\
\hline
\end{tabular}


Table 3. Comparison of the DT performance after pruning.

\begin{tabular}{lccccc}
\hline Alternative & $\begin{array}{c}\text { Sensivity } \\
(\mathrm{SE},(\%))\end{array}$ & $\begin{array}{r}\text { Specificity } \\
(\mathrm{SP},(\%))\end{array}$ & $\begin{array}{c}\text { Accuracy } \\
(\mathrm{ACC},(\%))\end{array}$ & $\begin{array}{c}\text { Number } \\
\text { of rules }\end{array}$ & $\begin{array}{c}\text { Number } \\
\text { of features }\end{array}$ \\
\hline 1 & 92.85 & 95.05 & 93.95 & 101 & 8 \\
2 & 91.80 & 94.75 & 93.28 & 38 & 8 \\
3 & 91.75 & 94.40 & 93.08 & 35 & 8 \\
4 & 92.50 & 93.60 & 93.05 & 30 & 8 \\
5 & 92.05 & 93.10 & 92.58 & 24 & 7 \\
\hline
\end{tabular}

The selection of the best tree was made taking into account the number of rules, the performance as well as the number of features. Comparing the results of 3 , it can be concluded that the fourth alternative is a suitable choice, as it presents a good compromise between the number of rules and the number of features. Given these results, this DT was considered acceptable to represent the GRACE score.

\subsection{Optimization}

In the optimization phase, the thresholds $(T)$ of DT were adjusted taking into account the PSC (set-1). A five-fold cross-validation was applied to assess how the results can be generalized. Five subsets were generated semi-randomly ensuring balanced subsets, with the same number of patients with event and no event (event rate approximately of 50\%), where four subsets were used as training set and the remaining was used as testing set. The algorithm was submitted to five iterations in order to test all the subsets combinations, being the optimization process applied to the rules that lead to wrong outputs.

The performance of the proposed approach depends of three variables ( $G, \beta$ and $\delta)$. In order to define their values, an exhaustive study was made testing the algorithms with different combinations of these variables. In particular, to each variable four different values were assigned: $G=1,0.1,0.01,0.001 ; \beta=0.2,0.3,0.4,0.5$; and $\delta=G$. Beside this, modifications in variable $\alpha$ were also introduced: $\alpha-\alpha \times$ $0.5, \alpha+\alpha \times 0.5$. The best results, accuracy values, are presented in Table 4 , being these results achieved through the average of the values obtained with the five datasets.

Table 4. Algorithms' performance according different combinations of variables $G, \beta, \delta$ and $\alpha$.

\begin{tabular}{|c|c|c|c|c|c|c|}
\hline$\alpha$ & $G$ & $\delta$ & $\beta$ & $\mathrm{SE}$ & SP & $\mathrm{ACC}$ \\
\hline \multicolumn{4}{|c|}{ Initial tree } & 89.64 & 60.15 & 61.33 \\
\hline$\alpha-\alpha \times 0.5$ & 0.001 & 0.001 & 0.4 & 84.26 & 74.43 & 79.34 \\
\hline$\alpha-\alpha \times 0.5$ & 0.01 & 0.01 & 0.4 & 80.33 & 77.70 & 79.02 \\
\hline$\alpha-\alpha \times 0.5$ & 0.1 & 1 & 0.3 & 84.59 & 73.11 & 78.85 \\
\hline \multirow[t]{2}{*}{$\alpha$} & 0.01 & 0.1 & 0.2 & 82.62 & 74.43 & 78.52 \\
\hline & & & GRACE & 90.82 & 50.16 & 70.49 \\
\hline
\end{tabular}


Table 5. Values of thresholds — initial and final values.

\begin{tabular}{lcrrrrrrrr}
\hline Index & $\begin{array}{c}\text { Risk } \\
\text { factor }\end{array}$ & Initial $T_{0}$ & Final $T$ & $\begin{array}{c}\text { Variation } \\
\%\end{array}$ & Index & $\begin{array}{c}\text { Risk } \\
\text { factor }\end{array}$ & $\begin{array}{c}\text { Initial } T_{0} \\
\text { Final } T\end{array}$ & $\begin{array}{c}\text { Variation } \\
\%\end{array}$ \\
\hline 1 & $x 1_{1}$ & 69.5 & 88.5 & 27.34 & 15 & $x 4_{1}$ & 3.06 & 4.28 & 39.87 \\
2 & $x 5_{1}$ & 1.5 & 3 & 100 & 16 & $x 7_{2}$ & 0.5 & 0.5 & 0 \\
3 & $x 3_{1}$ & 159.5 & 159.5 & 0 & 17 & $x 2_{1}$ & 110.5 & 103.5 & -6.35 \\
4 & $x 3_{2}$ & 119.5 & 118.5 & -0.83 & 18 & $x 5_{5}$ & 2.5 & 1.5 & -40.0 \\
5 & $x 3_{3}$ & 144.5 & 149.5 & 3.46 & 19 & $x 1_{5}$ & 61.5 & 46.5 & -24.39 \\
6 & $x 7_{1}$ & 0.5 & 0.5 & 0 & 20 & $x 3_{4}$ & 135.5 & 135.5 & 0 \\
7 & $x 5_{2}$ & 1.5 & 1.5 & 0 & 21 & $x 3_{5}$ & 130.5 & 130.5 & 0 \\
8 & $x 1_{2}$ & 60.5 & 59.5 & -1.65 & 22 & $x 4_{2}$ & 2.77 & 2.77 & 0 \\
9 & $x 6_{1}$ & 0.5 & 0.5 & 0 & 23 & $x 7_{3}$ & 0.5 & 0.5 & 0 \\
10 & $x 1_{3}$ & 49.5 & 49.5 & 0 & 24 & $x 1_{6}$ & 60.5 & 54.5 & -9.91 \\
11 & $x 5_{3}$ & 3.5 & 3.5 & 0 & 25 & $x 7_{4}$ & 0.5 & 0.5 & 0 \\
12 & $x 5_{4}$ & 1.5 & 1.5 & 0 & 26 & $x 1_{7}$ & 75.5 & 75.5 & 0 \\
13 & $x 8_{1}$ & 0.5 & 0.5 & 0 & 27 & $x 1_{8}$ & 82 & 82 & 0 \\
14 & $x 1_{4}$ & 82.5 & 82.5 & 0 & 28 & $x 3_{5}$ & 154 & 165.5 & 7.46 \\
& & & & & 29 & $x 4_{3}$ & 2.29 & 2.29 & 0 \\
\hline
\end{tabular}

The combination that is able to originate the best accuracy values $(79.34 \%)$ during the optimization procedure is alpha $-\alpha \times 0.5 ; G=0.001 ; \delta=0.001$ and $\beta=$ 0.4 (Table 4). Taking into account these parameters, the algorithm of optimization was applied to the whole dataset in order to find the new thresholds (Table 5) of the DT (Fig. 7).

As can be concluded from Table 5, during the optimization process the major variations in the threshold values occurred on the Killip class (index 2, variation $=100 \%$ ), (index 18, variation $=-40 \%$ ); heart rate (index 15, variation = $39.87 \%$ ); age (index 1, variation $=27.34 \%$ ), (index 19, variation $=-24.39 \%$ ), (index 24, variation $=-9.91 \%$; and systolic blood pressure (index 28, variation $=7.46 \%)$.

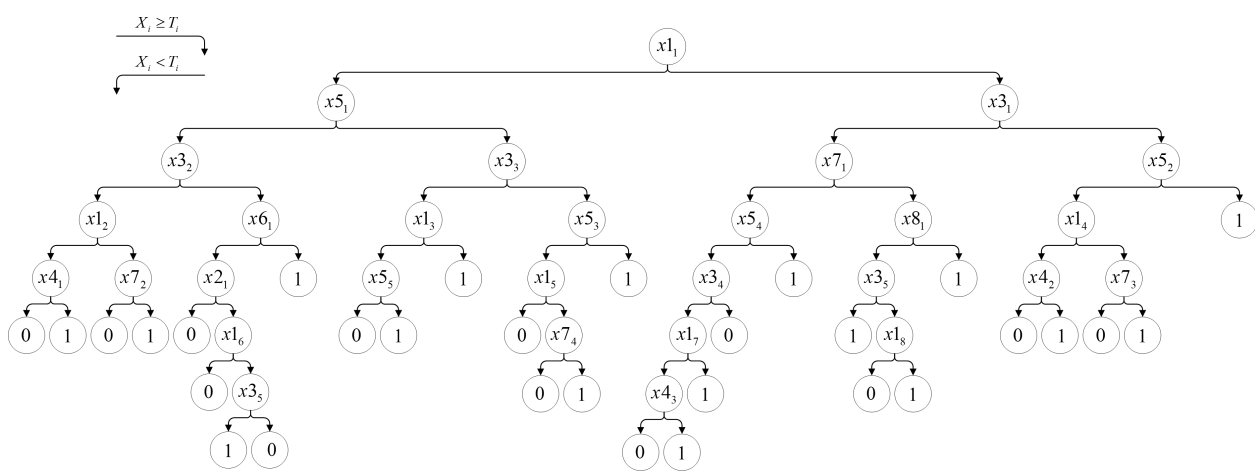

Fig. 7. Decision tree (best thresholds considering 30 rules). $x 1$ : age, $x 2$ : creatinine, $x 3$ : systolic blood pressure, $x 4$ : heart rate, $x 5$ : Killip class, $x 6$ : ST-segment deviation, $x 7$ : elevated cardiac markers, and $x 8$ : cardiac arrest at admission. 


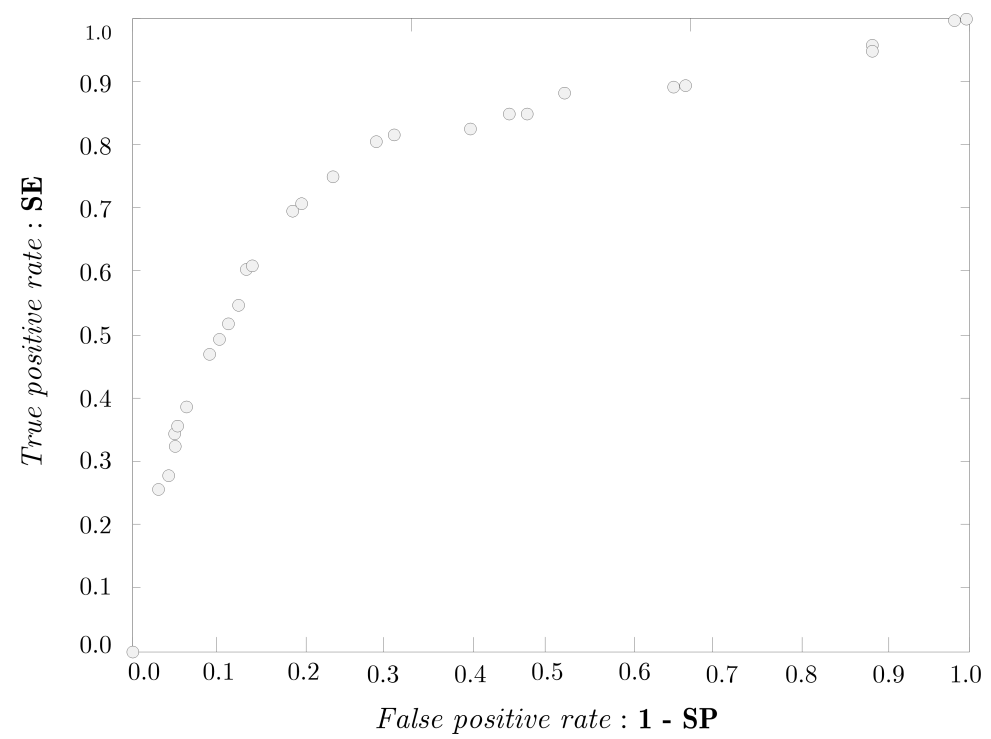

Fig. 8. ROC curve $-\mathrm{TH} \in[20 \ldots 50]$.

\subsection{Decision tree to new score}

In the last phase, a set of rules was derived, based on the GRACE score, and adjusted taking into account the dataset of the PSC. The next step of the proposed approach is the derivation of a new score based on the optimized DT. For each risk factor, the thresholds of the DT were organized in an ascending order and the respective ranges were created based on these values.

To find the threshold that best defines low risk and high risk patients, a ROC curve was derived (Fig. 8). Each point in the ROC curve corresponds to a threshold value in the range between 20 and 50, i.e., $\mathrm{TH} \in[20 \ldots 50]$. Although there are some points in the ROC curve that present acceptable results (Table 6), the value 34 is the threshold that leads to the best discrimination ability between low risk patients and high risk patients.

In Table 7, the performance between the new score and GRACE score can be compared. Additionally, to the ACC, the geometric mean (GM) was applied since

Table 6. Selected ROC curve values.

\begin{tabular}{lcccc}
\hline SE & SP & ACC & GM & Threshold \\
\hline 81.72 & 68.72 & 69.22 & 74.94 & 32 \\
80.65 & 70.77 & 71.15 & 75.55 & 33 \\
$\mathbf{7 5 . 2 7}$ & $\mathbf{7 6 . 0 5}$ & $\mathbf{7 6 . 0 2}$ & $\mathbf{7 5 . 6 6}$ & $\mathbf{3 4}$ \\
70.97 & 79.97 & 79.62 & 75.34 & 35 \\
69.89 & 80.98 & 80.55 & 75.23 & 36 \\
61.29 & 85.73 & 84.78 & 72.49 & 37 \\
\hline
\end{tabular}


Table 7. New score performance when applied to SPC set-2.

\begin{tabular}{lcccc}
\hline Mode & SE & SP & ACC & GM \\
\hline GRACE score & 86.81 & 52.04 & 53.38 & 67.21 \\
New score & 75.85 & 75.91 & 75.91 & 75.87
\end{tabular}

Table 8. Definition of new score.

\begin{tabular}{|c|c|c|c|c|}
\hline Risk factor & GRACE range & GRACE & New score range & New score \\
\hline Age & $\begin{array}{c}<40 \\
40-49 \\
50-59 \\
60-69 \\
70-79 \\
80-89 \\
>90\end{array}$ & $\begin{array}{c}0 \\
15 \\
29 \\
44 \\
59 \\
73 \\
80\end{array}$ & $\begin{array}{c}<59.5 \\
59.5-75.5 \\
75.5-82.5 \\
\geq 82.5\end{array}$ & $\begin{array}{c}1 \\
3 \\
6 \\
12\end{array}$ \\
\hline Systolic blood pressure & $\begin{array}{c}<80 \\
80-99 \\
100-119 \\
120-139 \\
140-159 \\
160-199 \\
>200\end{array}$ & $\begin{array}{c}57 \\
53 \\
43 \\
34 \\
24 \\
10 \\
0\end{array}$ & $\begin{array}{c}<118.5 \\
118.5-159.5 \\
\geq 159.5\end{array}$ & $\begin{array}{l}9 \\
3 \\
1\end{array}$ \\
\hline Killip class & $\begin{array}{l}1 \\
2 \\
3 \\
4\end{array}$ & $\begin{array}{c}0 \\
33 \\
67 \\
100\end{array}$ & $\begin{array}{l}1 \\
2 \\
3 \\
4\end{array}$ & $\begin{array}{c}2 \\
8 \\
14 \\
37\end{array}$ \\
\hline Heart rate & $\begin{array}{c}<70 \\
70-89 \\
90-109 \\
110-149 \\
150-199 \\
\geq 200\end{array}$ & $\begin{array}{c}0 \\
6 \\
12 \\
21 \\
32 \\
41\end{array}$ & $\begin{array}{l}<103.5 \\
\geq 103.5\end{array}$ & $\begin{array}{l}3 \\
8\end{array}$ \\
\hline Creatinine & $\begin{array}{c}0-0.39 \\
0.4-0.79 \\
0.8-1.19 \\
1.20-1.59 \\
1.6-1.99 \\
2.0-3.99 \\
\geq 4\end{array}$ & $\begin{array}{c}2 \\
5 \\
8 \\
11 \\
14 \\
23 \\
31\end{array}$ & $\begin{array}{l}<2.77 \\
\geq 2.77\end{array}$ & $\begin{array}{c}4 \\
12\end{array}$ \\
\hline Cardiac arrest at admission & $\begin{array}{l}0 \\
1\end{array}$ & $\begin{array}{c}0 \\
98\end{array}$ & $\begin{array}{l}0 \\
1\end{array}$ & $\begin{array}{c}4 \\
12\end{array}$ \\
\hline Elevated cardiac markers & $\begin{array}{l}0 \\
1\end{array}$ & $\begin{array}{c}0 \\
54\end{array}$ & $\begin{array}{l}0 \\
1\end{array}$ & $\begin{array}{l}2 \\
4\end{array}$ \\
\hline ST-segment deviation & $\begin{array}{l}0 \\
1\end{array}$ & $\begin{array}{c}0 \\
67\end{array}$ & $\begin{array}{l}0 \\
1\end{array}$ & $\begin{array}{l}2 \\
4\end{array}$ \\
\hline $\begin{array}{l}\text { Threshold } \\
\text { GRACE }\end{array}$ & $\begin{array}{c}\text { Non STE-ACS: } \\
\text { STE-ACS: }\end{array}$ & $\begin{array}{l}140 \\
154\end{array}$ & $\begin{array}{l}\text { Threshold } \\
\text { New score }\end{array}$ & 34 \\
\hline
\end{tabular}


the SPC set2 is not balanced and in these cases the GM is a more adequate metric than ACC.

The new score presents a lower sensitivity than the original GRACE score, but increases significantly the value of SP. As a result, the new score provides a much more balanced classifier as can be confirmed through the analysis of the ACC and GM values.

Finally, the score value attributed to each category is presented in Table 8. These scores were computed based on Eq. (16), the SPC set1 was used to derive the score and the set2 was used as the test set.

\section{Discussion}

The main motivation for this work is to develop a methodology to predict cardiovascular events capable of producing a better performance when compared with the risk assessment scores currently applied in the clinical practice. Moreover, if possible, the methodology should be simpler and produce a model that can be interpretable.

In order to create an interpretable model, a DT was used in the first phase. Using a pruning approach applied to the DT, a compromise between the number of nodes (interpretability) and the final performance was achieved. Moreover, to attain a structure similar to the current scores applied in clinical practice, the tree with optimized thresholds was converted into a score model. As can be observed in Table 7 , the new score presents a better balance between the sensitivity and specificity when compared with the GRACE score. Besides that, the new score also allowed the improvement of accuracy (approximately 22.53\%) and geometric mean $(8.66 \%)$ which represents a significant enhancement of the CVD risk prediction.

Additionally, in terms of applicability, the new score presents a simpler structure. As can be observed in Table 7, comparing the two scores (GRACE and the present one), the new score presents less categories. For example, the age risk score in the new approach is based on four different categories while the original GRACE model considers seven. Regarding heart rate and creatinine, the new approach uses two options while there are six and seven, respectively, in GRACE model. Finally, the systolic blood pressure risk factor has only three options while the original GRACE has seven options. These results (simpler structure) support the better interpretability of the model (when compared with GRACE).

In terms of adjustments to achieve these goals (better performance and simple structure) the major variation occurred on the Killip class, heart rate, age and systolic blood pressure (as depicted in Table 5). In fact, these risk factors are among the ones that contribute with a higher weight to the final score (Table 7). These adjustments have an important role in terms of performance, as can be concluded based on the sensitivity (initial tree: $89.64 \%$; optimized tree: $84.26 \%$ ) and the specificity (initial tree: $60.15 \%$; optimized tree: $74.43 \%$ ) values, Table 4 . Although the sensitivity suffers a smooth decrease (about $5 \%$ ), the specificity increased about $14 \%$. 


\section{Conclusions}

This work proposes a methodology to improve the performance exhibited by the prediction models applied in the current clinical practice based on information extracted from recent datasets. Three main phases were considered: (i) the development of a DT that can reproduce a score behavior (e.g., GRACE); (ii) the optimization of the derived DT, using recent datasets, through a new approach based on membership functions and finally (iii) the transformation of that DT into a score.

The first goal was achieved through the generation of a DT able to reproduce the GRACE score behavior. The second goal was reached by implementing an approach based on two specific functions: an error function, that gives information about the classification accuracy and a membership function which computes the degree of membership of each patient to a given condition in a node. Finally, the third step was addressed using the information of high risk patients rate in each one of the intervals defined.

In terms of structure, the new score presents less categories for each risk factor than the GRACE score. Besides this aspect, the new score is directly derived from a DT, thus ensuring the higher interpretability of the model (when compared with GRACE). Moreover, the obtained results show a rise in geometric mean of approximately $9 \%$ (from $67.21 \%$ to $75.87 \%$ ), which means that it is possible with this approach to obtain a more balanced classifier. In this perspective, it is possible to affirm that the new score performs better than the GRACE model for the PSC dataset. In fact, one of the biggest flaws of the original GRACE model was related with its poor specificity. The developed strategy, allowed an increase of this value, of approximately $23 \%$. Although an undesired effect in sensitivity value was also verified, this value decreased almost $11 \%$.

In upcoming work, the goal is to implement a methodology able to integrate information from several sources (knowledge driven and data driven approach) enhancing the personalization of risk prediction, and therefore creating a model with better performance to improve simultaneously the sensitivity and specificity values.

\section{Acknowledgments}

The authors would like to thank the Welcome FP7 611223 and LINK H2020 - 692023 projects and the PSC for their collaboration, in particular, in providing the clinical dataset.

\section{References}

1. T. A. Gaziano, K. S. Reddy, F. Paccaud and S. Horton, Cardiovascular disease, in Disease Control Priorities in Developing Countries, 2nd edn., Chapter 33 (The World Bank, 2006), doi: 10.1596/978-0-8213-6179-5.

2. World Health Organization, http://www.who.int/mediacentre/factsheets/fs317/en/ [accessed on 3rd March, 2016]. 
3. World Health Organization, http://www.who.int/nmh/countries/prt_en.pdf?ua=1 [accessed on 3rd March, 2016].

4. E. Ashley and J. Niebauer, Coronary artery disease, in Cardiology Explained (London: Remedica, 2004), pp. 1-2.

5. National Public Health Partnership, The Language of Prevention (Melbourne: NPHP, 2006), pp. 1-4.

6. P. De Araújo Gonçalves, J. Ferreira, C. Aguiar and R. Seabra-Gomes, TIMI, PURSUIT, and GRACE risk scores: Sustained prognostic value and interaction with revascularization in NSTE-ACS, Eur. Heart J. 26 (2005) 865-872.

7. S. Paredes, T. Rocha, P. de Carvalho, J. Henriques, M. Harris and J. Morais, Long term cardiovascular risk models' combination, Computer Methods and Programs in Biomedicine 101 (2011) 231-242, doi: 10.1016/j.cmpb.2010.12.015.

8. F. W. Verheugt, The GRACE registry: How real-life evidence contributes to acute coronary syndrome guidelines, European Heart Journal Supplements 17 (2015) D29-D31, doi: 10.1093/eurheartj/suv039.

9. M. de Mulder, T. van der Ploeg, G. A. de Waard, E. Boersma and V. A. Umans, Admission glucose does not improve GRACE score at 6 months and 5 years after myocardial infarction, Cardiology 120(4) (2011) 227-234, doi: 10.1159/000335715.

10. K. A. A. Fox, G. Fitzgerald, E. Puymirat, W. Huang, K. Carruthers, T. Simon, P. Coste, J. Monsegu, P. Gabriel Steg, N. Danchin and F. Anderson, Should patients with acute coronary disease be stratified for management according to their risk? Derivation, external validation and outcomes using the updated GRACE risk score, in BMJ Open 4(2) (2014) e004425, doi: 10.1136/bmjopen-2013-004425.

11. D. Mendes, S. Paredes, T. Rocha, P. Carvalho, T. Marques, J. Henriques and J. Morais, Short-term cardiovascular disease risk assessment: Comparison of two combination approaches, in 6th European Conf. Int. Federation for Medical and Biological Engineering Vol. 45 (Springer, Cham, 2015), pp. 670-673, doi: 10.1007/978-3-319-11128$5 \_167$.

12. Y. Peng, G. Kou, Y. Shi and Z. Chen, A descriptive framework for the field of data mining and knowledge discovery, International Journal of Information Technology \& Decision Making 7(4) (2007) 639-682, doi: 10.1142/S0219622008003204.

13. G. Kou, Y. Lu, Y. Peng and Y. Shi, Evaluation of classification algorithms using MCDM and rank correlation, International Journal of Information Technology 83 Decision Making 11(1) (2012) 197-225, doi: 10.1142/S0219622012500095.

14. G. Kou, Y. Peng and G. Wang, Evaluation of clustering algorithms for financial risk analysis using MCDM methods, Information Sciences 275 (2014) 1-12, doi: 10.1016/j. ins.2014.02.137.

15. G. Kou, D. Ergu, C. Lin and Y. Chen, Pairwise comparison matrix in multiple criteria decision making, Technological and Economic Development of Economy 22(5) (2016), pp. $738-765$, doi: $10.3846 / 20294913.2016 .1210694$.

16. D. Pal, C. Chakraborty and K. Mandana, Data mining approach for coronary artery disease screening, in International Conference on Image Information Processing, no. Iciip (IEEE, 2011), pp. 1-6, doi: 10.1109/ICIIP.2011.6108972.

17. M. R. Shouman, T. Turner and R. Stocker, Using decision tree for diagnosing heart disease patients, in Proc. Ninth Australasian Data Mining Conf. (2011), pp. 23-30.

18. L. Rokach and O. Maimon, Introduction to Decision Trees (World Scientific, Singapore, 2007), Vol. 69, pp. 1-11, doi: 10.1142/9789812771728_0001.

19. L. Rokach and O. Maimon, Data Mining and Knowledge Discovery Handbook (Springer, Boston, MA, 2010), doi: 10.1007/978-0-387-09823-4. 
20. L. Breiman, J. H. Friedman, R. A. Olshen and C. J. Stone, Classification and Regression Trees Chapter 1, 4 (Chapman, an. 1984).

21. R. J. Lewis, An introduction to classification and regression tree (CART) analysis, Annual Meeting of the Society for Academic Emergency Medicine (2000), doi: 10.1.1.95.4103.

22. H. H. Tung, C. Y. Chen, K. C. Lin, N. K. Chou, J. Y. Lee, D. L. Clinciu and R. Y. Lien, Classification and regression tree analysis in acute coronary syndrome patients, World Journal of Cardiovascular Diseases 2(3) (2012) 177-183, doi: 10.4236/wjcd. 2012.23030.

23. L. Rokach and O. Maimon, Splitting criteria, in Data Mining with Decision Trees (World Scientific, 2007), pp. 53-61, doi:10.1007/978-0-387-09823-4.

24. S. Sivagama Sundhari, A knowledge discovery using decision tree by Gini coefficient, in Int. Conf. Business, Engineering and Industrial Applications (IEEE, 2011), pp. 232235, doi: 10.1109/ICBEIA.2011.5994250.

25. J. Hu, Y. Wang, E. Zhou, M. C. Fu and S. I. Marcus, A survey of some model-based methods for global optimization, in Optimization, Control, and Applications of Stochastic Systems (Birkhäuser, Boston, 2012), pp. 157-179, doi: 10.1007/978-0-8176-8337-5_10.

26. Y. Zhang and Y.-J. Lv, Optimize algorithm of decision tree based on rough sets hierarchical attributes, in Int. Conf. Computational Intelligence and Security (IEEE, 2008), pp. 133-137, doi: 10.1109/CIS.2008.89.

27. H. Yang and S. Fong, Incremental optimization mechanism for constructing a decision tree in data stream mining, Mathematical Problems in Engineering 2013 (2013) 1-14, doi: $10.1155 / 2013 / 580397$.

28. W. Yi, J. Duan and M. Lu, Optimization of decision tree based on variable precision rough set, in Int. Conf. Artificial Intelligence and Computational Intelligence, Vol. 3 (IEEE, 2010), pp. 148-151, doi: 10.1109/AICI.2010.270.

29. P. J. García-Laencina, J.-L. Sancho-Gómez and A. R. Figueiras-Vidal, Pattern classification with missing data: A review, Neural Computing and Applications 19 (2010) 263-282, doi: 10.1007/s00521-009-0295-6.

30. T. G. Investigators, https://www.outcomes-umassmed.org/grace/files/GRACE_Risk Model_Coefficients.pdf [accessed on 3rd March, 2016].

31. L. Paiva, R. Providência, S. N. Barra, P. Dinis, A. C. Faustino, M. Costa and L. Gonçalves, Improving risk stratification in non-ST-segment elevation myocardial infarction with combined assessment of GRACE and CRUSADE risk scores, Archives of Cardiovascular Diseases 107 (2014) 681-689.

32. Calculator GRACE score, https://www.outcomes-umassmed.org/grace/acs_risk/acs_ risk_content.html [accessed on 3rd March, 2016].

33. Risk table GRACE score, https://www.outcomes-umassmed.org/grace/grace_risk_table. aspx [accessed on 3rd March, 2016]. 